\title{
RISCO DE CONTAMINAÇÃO POR AGROTÓXICOS DAS ÁGUAS SUBTERRÂNEAS EM ÁREAS CULTIVADAS COM CANA-DE-AÇÚCAR
}

RAFAELA RUY*

TERESINHA ESTEVES DA SILVEIRA REIS**

\begin{abstract}
Realizou-se a simulação da lixiviação de agrotóxicos utilizados na cultura da cana-de-açúcar no município de Bandeirantes (PR), com auxílio do programa Análise de Risco Ambiental de Agrotóxicos (ARAQuá), desenvolvido pela EMBRAPA. Utilizou-se o mapa de solos do Município para selecionar três propriedades da Usina de Bandeirantes, nas quais foram coletados dados sobre a aplicação de agrotóxicos durante o ano de 2010. As características dos solos desses locais e as propriedades dos agrotóxicos utilizados foram pesquisadas na literatura, efetuando-se sua inclusão no programa. Após a criação de 12 diferentes cenários, combinando os agrotóxicos utilizados com os solos, foram gerados relatórios para informar possível contaminação da água subterrânea pela lixiviação desses compostos. Usou-se a toxicidade dos produtos para plantas aquáticas, grupo mais afetado pela presença de herbicidas, como referência para verificar a contaminação da água. Dentre os agrotóxicos aplicados naquele ano, somente quatro apresentaram potencial de contaminação de águas subterrâneas nas condições estabelecidas: hexazinona, diuron, tebutiron e sulfentrazona na Fazenda Goivira; e diuron e hexazinona na Fazenda Paraguay.
\end{abstract}

PALAVRAS-CHAVE: ÁGUAS SUBTERRÂNEAS - CONTAMINAÇÃO; LIXIVIAÇÃO; HERBICIDAS; CANA-DEAÇÚCAR.

* Bióloga, Universidade Estadual do Norte do Paraná, Campus Luiz Meneghel, Bandeirantes, PR (e-mail: rafaelaruy@cnpso.embrapa.br).

** Engenheira Agrônoma, Professora, Universidade Estadual do Norte do Paraná, Campus Luiz Meneghel. Bandeirantes, PR (e-mail: tsreis@uenp.edu.br). 


\section{INTRODUÇÃO}

A demanda mundial crescente por fontes alternativas na produção de energia tem suscitado debate acerca de seus impactos ambientais. No âmbito internacional, maior consciência sobre a relação entre o uso de combustíveis fósseis e as mudanças climáticas incentivou a procura pelo etanol combustível em razão de seus efeitos positivos na absorção de $\mathrm{CO}_{2}$. No Brasil, o aumento das vendas de carros bicombustíveis impulsionou a demanda pelo álcool hidratado, ocasionando reversão da tendência declinante do seu uso até meados de 2003 (MORAES, 2007).

Com o avanço do uso do etanol como combustível houve crescimento na área cultivada com cana-de-açúcar, que no Brasil é a matéria-prima para a produção do etanol. Esse aumento na produção da cultura da cana-de-açúcar, conforme Barbosa (2007), pode provocar impactos devido ao uso de agrotóxicos que levam à contaminação de rios, aquíferos, nascentes e do solo.

Para que a expansão do plantio de cana viesse a favorecer a população sem detrimento do meio ambiente e da economia nacional foi lançado o zoneamento ecológico da cana. O norte do Paraná, região em que se encontra o município de Bandeirantes, apresenta alta aptidão agrícola, conforme o zoneamento ecológico-econômico proposto por Manzato et al. (2009). No entanto, a ampliação da área plantada com cana-de-açúcar traz como consequência à elevação do consumo de agrotóxicos usados na cultura. $O$ risco de ocorrência de agrotóxicos na água subterrânea evidencia a necessidade de estudos sobre os processos de transporte desses compostos, que devem enfocar os processos de lixiviação e de escoamento superficial (CEDERNEIRA et al., 2005a).

As práticas agrícolas são particularmente importantes para a poluição, devido à constante liberação de agrotóxicos no ambiente, cujos riscos de contaminação ambiental são agravados pela falta de acompanhamento técnico e sua utilização em locais inadequados, como sítios de recarga de aquíferos (HENN, 2009).

O uso frequente, e muitas vezes incorreto, de agrotóxicos pode oferecer ameaça de contaminação das águas superficiais e subterrâneas (SPADOTTO et al., 2010). Pesquisas realizadas em todo o mundo constataram o alarmante crescimento dos registros de contaminação de águas destinadas ao abastecimento, como decorrência da utilização intensiva e acúmulo de compostos com alto grau de toxicidade e persistência (HENN, 2009)

A maioria dos solos apresenta-se como a mistura de subtipos, com características complexas. Dessa forma, observa-se amplo espectro de características químicas associadas à ligação ou adesão de cada contaminante, proporcionando mecanismos diferentes ou interação com cada tipo de solo (TAKAKI, OKADA e NASCIMENTO, 2006). Assim, os processos relacionados a esses fenômenos devem ser detalhadamente estudados para se verificar como ocorre o transporte dessas moléculas através do solo (BARIZON et al., 2006).

A Embrapa Meio Ambiente desenvolveu o software Avaliação de Risco Ambiental por Agrotóxico (ARAquá) para auxiliar as análises de riscos ambientais, considerando as possíveis contaminações de corpos d'água subterrâneos, mediante comparação de suas concentrações estimadas em cenários de uso agrícola com parâmetros de qualidade da água (SPADOTTO et al., 2010).

Este estudo teve como objetivo avaliar o risco ambiental de lixiviação dos agrotóxicos utilizados na cultura de cana-de-açúcar, no município de Bandeirantes (PR), mediante simulação efetuada pelo programa ARAquá.

\section{MATERIAL E MÉTODOS}

Foram criados cenários pela inserção dos seguintes dados em campos específicos do programa ARAquá: 
- caracterização do local - precipitação (mm/ano); irrigação (mm/ano); evapotranspiração $(\mathrm{mm} /$ ano); porosidade (v/v);

- caracterização do solo - profundidade $(\mathrm{cm})$; capacidade de campo $(\mathrm{v} / \mathrm{v})$; densidade $\left(\mathrm{g} / \mathrm{cm}^{3}\right)$; carbono orgânico (v/v);

- propriedades dos agrotóxicos - ingrediente ativo; dose; padrão de potabilidade ( $\mu \mathrm{g} / \mathrm{L})$; toxicidade para organismos aquáticos $(\mu \mathrm{g} / \mathrm{L})$; coeficiente de sorção - Koc $(\mathrm{mL} / \mathrm{g})$ e meia-vida.

\subsection{CARACTERIZAÇÃO DO LOCAL}

O município de Bandeirantes (PR) localiza-se nas coordenadas 50²9'44,08” - 5009'42,56" W e $23^{\circ} 17{ }^{\prime} 5.10^{\prime \prime}-23^{\circ} 00^{\prime} 58.40^{\prime \prime} \mathrm{S}$, apresentando precipitação média anual de $1.300 \mathrm{~mm}$ (LIMA, 2007). Calculou-se a evapotranspiração no referido município utilizando os dados de Abumanssur (2006), que resultou na média de $86 \mathrm{~mm} / \mathrm{mês}$. Dados que representam as médias da profundidade e da porosidade dos solos em estudo foram empregados para todos os cenários. A profundidade média utilizada foi de $200 \mathrm{~cm}$ e a porosidade de 49\% (EMBRAPA, 1984).

\subsection{CARACTERIZAÇÃO DOS TIPOS DE SOLO}

O município de Bandeirantes apresenta solos muito homogêneos em virtude da uniformidade geológica e à pequena variação climática, encontrando-se extensas áreas com o mesmo padrão pedológico (REIS, BARROS e REIS, 2004). Os solos da região do município foram classificados, conforme levantamento realizado pela Embrapa (1999), e distribuídos em mapa de solos (Figura 1). As proporções de cada tipo de solo por área $\left(\mathrm{em} \mathrm{Km}^{2}\right)$ no município de Bandeirantes foram calculadas por Reis, Barros e Reis (2004) como: Latossolo Vermelho Eutroférrico 10,1\%, Latossolo Vermelho Eutroférrico e Nitossolo Eutroférrico 1,7\%, Nitossolo Eutroférrico $62,4 \%$, Chernossolo + Neossolo Litólico 15,1\% e Chernossolo + Neossolo Litólico + Nitossolo Eutroférrico $10,6 \%$.

Realizou-se a sobreposição dos mapas de solos do município e das propriedades em que a Usina Bandeirantes produz cana-de-açúcar, considerando as informações completas e registradas dos agrotóxicos aplicados nesses locais. Fazendas com tipos diferentes de solo foram escolhidas, recolhendo-se os dados a respeito dos agrotóxicos utilizados em 2010.

Foram selecionadas para o estudo a Fazenda Paraguay, com solo do tipo Latossolo Vermelho Eutroférrico; a Fazenda São Carlos com solo do tipo Nitossolo Eutroférrico e a Fazenda Goivira que apresenta solo do tipo Associação Brunizem Avermelhado + Solos Litólicos eutrófico + Terra Roxa Estruturada eutrófica.

A Fazenda Goivira, situada em região de solo do tipo Associação (EMBRAPA, 1999), foi considerada para a base de estudos como local de transição entre o Nitossolo Eutroférrico e o Latossolo Vermelho Eutroférrico. Os solos Litólicos e Brunizem, apesar de contidos na região em macro escala, não estão presentes nas áreas de cultivo de cana (pois são típicos de regiões com inclinação, o que impossibilita a cultura).

Os dados referentes aos tipos de solo em estudo estão discriminados na Tabela 1.

\subsection{CARACTERIZAÇÃO DOS AGROTÓXICOS}

Os dados de aplicação e dosagem dos produtos foram obtidos no acervo da Usina Bandeirantes e os dos princípios ativos nas respectivas notas técnicas.

Os agrotóxicos utilizados, com nome comercial e princípio ativo constam na Tabela 2, assim como, os dados de meia-vida e parâmetros para organismos aquáticos obtidos na base de dados da IUPAC (2011). 


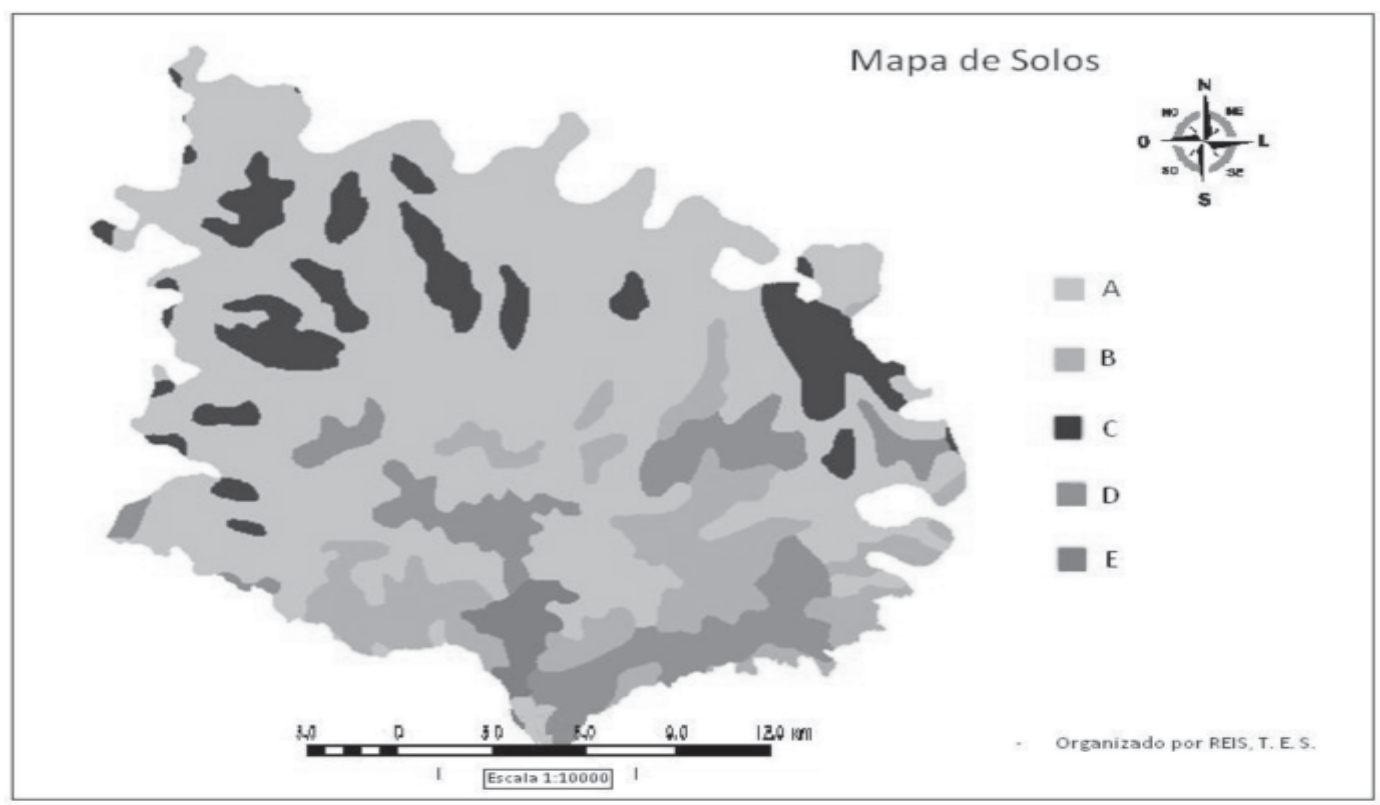

FIGURA 1 - UNIDADES E ASSOCIAÇÕES DE SOLOS OBSERVADAS NO MUNICÍPIO DE BANDEIRANTES (PR)

A) Nitossolo Eutroférrico; B) Bv2: associação de Chernossolos e Neossolos Litólicos; C) Latossolo Vermelho Eutroférrico; D) associação de Chernossolos, Neossolos Litólicos e Nitossolo Eutroférrico e E) Latossolo Vermelho Eutroférrico e Nitossolo Eutroférrico.

TABELA 1 - PERFIS DO LATOSSOLO, NITOSSOLO E ASSOCIAÇÃO

\begin{tabular}{|c|c|c|c|c|}
\hline Solo & Camadas & $\begin{array}{l}\text { Profundidade } \\
\quad(\mathrm{cm})\end{array}$ & $\begin{array}{l}\text { Densidade do solo } \\
\qquad\left(\mathrm{g} / \mathrm{cm}^{3}\right)\end{array}$ & $\begin{array}{c}\text { Carbono orgânico } \\
(\%)\end{array}$ \\
\hline \multirow{4}{*}{ Latossolo } & Ap & 21 & 1,19 & 1,45 \\
\hline & B1 & 38 & 1,11 & 0,73 \\
\hline & B21 & 87 & 1,02 & 0,60 \\
\hline & B22 & 190 & 1,02 & 0,32 \\
\hline \multirow{4}{*}{ Nitossolo } & Ap & 15 & 1,19 & 2,49 \\
\hline & B1t & 32 & 1,26 & 1,08 \\
\hline & B21t & 74 & 1,20 & 0,78 \\
\hline & B22t & 154 & 1,25 & 0,41 \\
\hline \multirow{4}{*}{$\begin{array}{c}\text { Associação } \\
\text { Latossolo } \\
+ \\
\text { Nitossolo }\end{array}$} & Ap & 18 & 1,19 & 1,97 \\
\hline & B1 & 35 & 1,185 & 0,905 \\
\hline & B21 & 80,5 & 1,11 & 0,69 \\
\hline & B22 & 172 & 1,135 & 0,365 \\
\hline
\end{tabular}

Fonte: EMBRAPA (1984).

Segundo Callisto, Gonsalves Jr e Moreno (2005), o uso de bioindicadores de qualidade de água exige o conhecimento das comunidades biológicas que devem ser monitoradas em 
determinado ecossistema. Considerando que todos os agrotóxicos utilizados nas áreas estudadas são herbicidas, elegeu-se a toxicidade dos produtos para plantas aquáticas como parâmetro para organismos aquáticos.

As plantas aquáticas são produtoras primárias do ecossistema, portanto a perda do primeiro nível trófico afetará direta ou indiretamente todas as outras espécies.

Foram utilizadas plantas aquáticas do gênero Lemna, sendo analisados os efeitos agudos da concentração eficiente (EC50) dos produtos no período de 7 dias (Tabela 2).

\section{TABELA 2 - DOSAGENS DOS AGROTÓXICOS POR PROPRIEDADE E RESPECTIVOS ATRIBUTOS QUÍMICOS}

\begin{tabular}{|c|c|c|c|c|c|c|c|c|}
\hline Propriedade & Solo & $\begin{array}{c}\text { Nome } \\
\text { Comercial }\end{array}$ & $\begin{array}{l}\text { Dose } \\
\text { (g/ha) }\end{array}$ & $\begin{array}{c}\text { Ingrediente } \\
\text { ativo } \\
(\%)\end{array}$ & $\begin{array}{l}\text { Dose do } \\
\text { Princípio } \\
\text { ativo } \\
\text { (g/ha) }\end{array}$ & $\begin{array}{l}\text { Meia } \\
\text { vida } \\
\text { (dia) }\end{array}$ & $\begin{array}{c}\mathrm{Koc} \\
(\mathrm{mL} / \mathrm{g})\end{array}$ & $\begin{array}{c}\text { Parâmetros } \\
\text { para plantas } \\
\text { aquáticas } \\
(\mu \mathrm{g} / \mathrm{L})\end{array}$ \\
\hline \multirow{3}{*}{ Paraguay } & \multirow{3}{*}{ Latossolo } & Herbipak® & 3000 & $\begin{array}{c}\text { Ametrina } \\
46,16 \%\end{array}$ & 1384,8 & 37 & 316 & 10 \\
\hline & & \multirow{2}{*}{ Hexaron $®$} & \multirow{2}{*}{2500} & $\begin{array}{c}\text { Diuron } \\
46 \% \\
\end{array}$ & 1150 & 75,5 & 813 & 18,3 \\
\hline & & & & $\begin{array}{c}\text { Hexazinona } \\
13,2 \%\end{array}$ & 330 & 105 & 54 & 72 \\
\hline \multirow{4}{*}{ São Carlos } & \multirow{4}{*}{ Nitossolo } & Aminol® & 2000 & $\begin{array}{c}2,4-\mathrm{D} \\
42,30 \%\end{array}$ & 846 & 10 & 88,4 & 580 \\
\hline & & Herbipak® & 4000 & $\begin{array}{c}\text { Ametrina } \\
46,16 \%\end{array}$ & 1846,4 & 400 & 80 & 135 \\
\hline & & \multirow{2}{*}{ Velpar® } & \multirow{2}{*}{2000} & $\begin{array}{c}\text { Hexazinona } \\
17,35 \% \\
\end{array}$ & 347 & 105 & 54 & 72 \\
\hline & & & & $\begin{array}{c}\text { Diuron } \\
61,52 \% \\
\end{array}$ & 1230,4 & 75,5 & 813 & 18,3 \\
\hline \multirow{5}{*}{ Goivira } & \multirow{5}{*}{ Associação } & Butiron® & 1500 & $\begin{array}{c}\text { Tebutiron } \\
44,55 \%\end{array}$ & 668,25 & 400 & 80 & 135 \\
\hline & & DMA® & 500 & $\begin{array}{c}2,4-D \\
42,62 \%\end{array}$ & 213,1 & 10 & 316 & 580 \\
\hline & & \multirow{2}{*}{ Velpar® } & \multirow{2}{*}{2000} & $\begin{array}{c}\text { Hexazinona } \\
17.35 \%\end{array}$ & 347 & 105 & 54 & 72 \\
\hline & & & & $\begin{array}{l}\text { Diuron } \\
61,52 \%\end{array}$ & 1230,4 & 75,5 & 813 & 18,3 \\
\hline & & Boral ${ }^{\circledR}$ & 1500 & $\begin{array}{c}\text { Sulfentrazona } \\
40,95 \%\end{array}$ & 614,25 & 541 & 43 & 290 \\
\hline
\end{tabular}

Fonte: DOW AGROSCIENCE..., 2008; DU PONT..., 2008; FMC..., 2008; MILÊNIA AGROCIÊNCIAS, 2002, 2006, 2009, 2010.

Após a inserção dos dados nos seus respectivos campos, o programa permite o cálculo da lixiviação e a geração de relatórios. Cada relatório tem como base o princípio ativo e não o herbicida, entretanto o mesmo herbicida pode apresentar ação combinada de dois princípios ativos. Assim, a dosagem do herbicida foi calculada de acordo com a proporção do princípio ativo no produto, (Tabela 2).

\section{RESULTADOS E DISCUSSÃO}

Cada cenário propiciou a geração de relatórios contendo a quantidade de agrotóxico que sofreu lixiviação e que se espera encontrar nas águas subterrâneas (Tabela 3).

Na Fazenda Paraguay, os princípios ativos ametrina e diuron nas quantidades utilizadas não se mostraram potencialmente poluidores da água por lixiviação na simulação realizada.

A hexazinona apresentou perda de 4,53 E-249 $\mu \mathrm{g}$.i.a/L por lixiviação até o topo do aquífero, com concentração estimada nas águas subterrâneas de 1,50 E-247 $\mu$ g.i.a/L. O parâmetro para 
organismos aquáticos estimado para hexazinona foi de $72 \mu \mathrm{g} / \mathrm{L}$.

Dos princípios ativos utilizados na Fazenda São Carlos, conforme a Tabela 3, os herbicidas ametrina, hexazinona, diuron e 2,4-D não ofereceram risco de contaminação das águas subterrâneas nas condições empregadas.

\section{TABELA 3 - RESULTADOS DOS RELATÓRIOS GERADOS PELO PROGRAMA ARAQUá}

\begin{tabular}{|c|c|c|c|c|}
\hline Fazenda & Ingrediente ativo & $\begin{array}{c}\text { Concentração estimada } \\
\text { nas águas subterrâneas } \\
(\mu \mathrm{g} / \mathrm{L})\end{array}$ & $\begin{array}{c}\text { Perda por } \\
\text { lixiviação até o } \\
\text { topo do aquífero } \\
(\mu \mathrm{g} / \mathrm{L})\end{array}$ & $\begin{array}{l}\text { Parâmetro } \\
\text { para plantas } \\
\text { aquáticas } \\
(\mu \mathrm{g} / \mathrm{L})\end{array}$ \\
\hline \multirow{3}{*}{ Paraguay } & Ametrina & 0,00 & 0,00 & 10 \\
\hline & Diuron & 0,00 & 0,00 & 18,3 \\
\hline & Hexazinona & $1,50 \mathrm{E}-247$ & $4,53 \mathrm{E}-249$ & 72 \\
\hline \multirow{4}{*}{ São Carlos } & $2,4-D$ & 0,00 & 0,00 & 580 \\
\hline & Ametrina & 0,00 & 0,00 & 10 \\
\hline & Hexazinona & 0,00 & 0,00 & 72 \\
\hline & Diuron & 0,00 & 0,00 & 18,3 \\
\hline \multirow{5}{*}{ Goivira } & Tebutiuron & $3,61 \mathrm{E}-118$ & $5,37 \mathrm{E}-120$ & 135 \\
\hline & $2,4-D$ & 0,00 & 0,00 & 580 \\
\hline & Hexazinona & 1,36 E-306 & 3,90 E-308 & 72 \\
\hline & Diuron & 0,00 & 0,00 & 18,3 \\
\hline & Sulfentrazona & 1,56 E-46 & $2,53 \mathrm{E}-48$ & 290 \\
\hline
\end{tabular}

Na Fazenda Goivira, o 2,4-D e o diuron não revelaram potencial poluidor para as águas de acordo com a simulação efetuada. Já a hexazinona, o tebutiuron e a sulfentrazona apresentaram lixiviação para a água subterrânea, perdendo-se 3,90 E-308 $\mu \mathrm{g} / \mathrm{L}$ de hexazinona até o topo do aquífero com concentração de 1,36 E-306 $\mu \mathrm{g} / \mathrm{L}$ nas águas subterrâneas. O tebutiuron apresentou concentração estimada de 3,61 E-118 $\mu \mathrm{g} / \mathrm{L}$ nas águas subterrâneas com perda por lixiviação até o topo do aquífero de $5,37 \mathrm{E}-120 \mu \mathrm{g} / \mathrm{L}$. O parâmetro para organismos aquáticos (135 $\mu \mathrm{g} / \mathrm{L}$ ), não revelou risco para as plantas aquáticas na quantidade utilizada. A sulfentrazona também demonstrou potencial poluidor, sendo a sua concentração estimada nas águas subterrâneas de 1,56 E-46 $\mu \mathrm{g} / \mathrm{L}$ e o total perdido por lixiviação até o topo do aquífero de 2,53 E-48 $\mu \mathrm{g} / \mathrm{L}$.

Na Fazenda Goivira e na Fazenda Paraguay foram utilizados agrotóxicos em condições que ofereceram risco ambiental, segundo o programa ARAQuá, pois resultaram na lixiviação dos produtos para as águas subterrâneas.

Nenhuma das fazendas, isoladamente, causou poluição suficiente das águas para afetar as plantas aquáticas (Tabela 3 ).

A Hexazinona mostrou maior potencial poluidor de águas subterrâneas em Latossolo do que em Nitossolo. Sua aplicação em menor quantidade (que na Fazenda São Carlos) foi capaz de causar lixiviação na Fazenda Paraguay.

Deve-se considerar que a degradabilidade dos agrotóxicos foi testada em condições ideais de temperatura e pH e em localidade diferente da área em estudo. As condições climáticas reais, assim como os tipos de micro-organismos do solo, podem afetar o tempo de degradação dos produtos.

Milhome et al. (2009) empregaram os métodos screening e o índice de vulnerabilidade de águas subterrâneas (GUS) para analisar o potencial de contaminação do 2,4-D. O produto foi considerado, segundo o método screening como contaminador potencial e pelo índice de GUS como faixa de transição, sendo classificado pelos autores com contaminador potencial.

Armas et al. (2005), utilizando os cálculos de GUS e Leachability Index - LEACH (log 10) 
evidenciaram o alto potencial de lixiviação dos herbicidas tebutiurom e sulfentrazona, classificados pelos autores como produtos de alta mobilidade e toxicidade. De todos os princípios ativos pesquisados em ambos os estudos, esses produtos alcançaram os maiores índices de GUS e LEACH ao mesmo tempo. Outros princípios ativos apresentaram grande potencial de lixiviação somente para o GUS (ametrina) ou para o LEACH (hexazinona), porém Armas et al. (2005) não relacionaram os agrotóxicos aos diferentes tipos de solo em que foram aplicados.

Cederneira et al. (2005b) não constataram a presença de herbicidas da classe das triazinas em áreas de recarga de aquífero em razão desse tipo de produto não ultrapassar $20 \mathrm{~cm}$ de profundidade no solo. $O$ solo na região de Bandeirantes (PR) é profundo e essa característica influenciou os resultados, pois a ametrina (classe das atrazinas) não apresentou risco de lixiviação para a água subterrânea de acordo com o programa utilizado.

Rosa, Pinheiro e Silva (2007), em estudo comparativo dos tipos de agrotóxicos verificaram maior potencial de lixiviação dos herbicidas, seguidos pelos inseticidas e fungicidas. Armas et al. (2005) mostraram a importância dos estudos abrangendo os herbicidas, que compõem a classe de agrotóxicos mais utilizada na cultura de cana-de-açúcar.

\section{CONCLUSÃO}

A mobilidade dos agrotóxicos depende tanto do meio no qual o produto foi inserido, quanto das suas características químicas e físicas.

Verificou-se menor contaminação em Nitossolo, pois sendo mais argiloso proporciona maior sorção do herbicida.

Os herbicidas com meia-vida mais longa e valores menores de Koc mostraram risco maior de contaminação de águas subterrâneas.

Hexazinona, tebutiuron e sulfentrazona apresentaram lixiviação nas condições estudadas, mas a quantidade dos produtos que atinge as águas subterrâneas é insuficiente para causar toxicidade às plantas aquáticas. No entanto, o efeito cumulativo considerando todas as propriedades da região pode ampliar o risco de contaminação das águas subterrâneas.

\section{ABSTRACT \\ AGROCHEMICALS RISK OF CONTAMINATION OF GROUNDWATER IN CULTIVATED AREAS WITH SUGAR CANE}

In the present work a simulation of the agrochemicals lixiviation used in the culture of sugar cane in the city of Bandeirantes (Paraná-Brazil) was performed using the Environmental Risk Analysis of Pesticides (ARAQuá), developed by EMBRAPA. Using the soil map of the city, three properties from the Bandeirantes sugar mill were selected for collecting data on chemical use during the year of 2010. Literature review was performed for the characteristics of the soil from these areas and the properties of the utilized pesticides, and data were entered into the program. After the development of 12 different scenarios, combining the pesticides and soil, reports were generated to inform a possible contamination of groundwater by lixiviation of these agrochemicals. The toxicity over aquatic plants, the most affected group by the presence of herbicides, was used as a parameter to verify the contamination of the water. Among the products utilized on that year, only four presented groundwater contamination potential in the studied conditions: hexazinone, diuron, tebutiron and sulfentrazone, on the Goivira farm; and diuron and hexazinone in Paraguay farm.

KEY-WORDS: GROUNDWATER-CONTAMINATION; LIXIVIATION; HERBICIDES; SUGAR CANE.

\section{REFERÊNCIAS}

1 ABUMANSSUR, C. Estimativa da evapotranspiração mensal no Estado do Paraná. 2006. 102 f. Dissertação (Mestrado em Engenharia Agrícola), Universidade Estadual do Oeste do Paraná, Cascavel, 2006.

2 ARMAS, E. D. et al. Uso de agrotóxicos em cana-de-açúcar na bacia do rio Corumbataí e risco de contaminação de poluição hídrica. Química Nova, v.28, n. 6. p. 975-982, junho 2005.

3 BARBOSA, L.M. Agroenergia, biodiversidade, segurança alimentar e direitos humanos. Conjuntura Internacional, v. 4, n. 33, p. 1-6, 2007. 
4 BARIZON, R.R.M.; LAVORENTI, A.; REGITANO, J.B.; PRATA, F.; TORNISIELO, V.L. Simulação do transporte e da sorção de imazaquin em colunas de solo. R. Brasileira de Ciência do Solo, v. 30, p.615-623, 2006.

5 CALLISTO, M.; GONSALVES JUNIOR, J. F.; MORENO, P. Invertebrados aquáticos como bioindicadores. Universidade Federal de Minas Gerais, Belo Horizonte, 2005. Disponível em: <http//www.icb.ufmg.br/ bentos> Acesso em: 10 agosto de 2011.

6 CEDERNEIRA, A. L; SPADOTTO, C. A.; MATALLO, M. B.; GOMES, M. A. F. Sorção do herbicida 2,4-D em solos brasileiros. Pesticidas: revista de ecotoxicologia e meio ambiente, Curitiba, v.13, p.103-110, jan./dez. 2005a.

7 CEDERNEIRA, A.L.; SOUZA, M.D.; PESSOA, M.C.P.Y.; QUEIROZ, S.C.N.; FERRACINI, V.L.; LANCHOTE, V.L.; QUEIROZ, R.H.C.; BOLONHEZI, D.; FARJANI NETO, C. Efeito de sistemas de cultivo com cana-de-açúcar sobre a lixiviação dos herbicidas do grupo das triazinas em região de recarga do Aquífero Guarani. Jaguariúna-SP: Embrapa Solos, 2005b. (Boletim de Pesquisa e Desenvolvimento, 32).

8 DOW AGROSCIENCE INDUSTRIAL LTDA. DMA 806 BR. São Paulo, 2008. 6 p.

9 DU PONT DO BRASIL S.A. VELPAR MAX. Rio de Janeiro, 2008. 8 p.

10 EMBRAPA. Centro Nacional de Pesquisa de Solo. Sistema Brasileiro de classificação de solos. Rio de Janeiro, 1999. $412 \mathrm{p}$.

11 EMBRAPA. Serviço Nacional de Levantamento e Conservação de Solos. Levantamento de reconhecimento dos solos do Estado do Paraná. Curitiba, 1984. 414 p.

12 FMC QUÍMICA DO BRASIL. BORAL 500 S. Uberaba, 2008.14 p.

13 HENN, C. Seleção de linhagens de basidiomicetos resistentes aos herbicidas atrazina e diuron: produção de enzimas lignolíticas e degradação dos compostos. 2009. 110 f. Dissertação (Mestrado em Microbiologia Aplicada) Universidade Estadual Paulista, São José do Rio Preto, 2009.

14 International Union of Pure and Applied Chemistry (IUPAC). Pesticide Properties Database (PPDB). Disponível em : <http://sitem.herts.ac.uk/aeru/footprint/es/Reports/601.htm\#trans>Acesso em: 13/08/11

15 LIMA, C.B.; BELLETTINI, N.M.T.; SILVA, A.S.; JANANE, J.K.; AMADOR, T.S.; VIEIRA, M.A.V.; CHEIRUBIM, A.P. Descrição das árvores encontradas nas ruas de Bandeirantes (PR). Revista Brasileira de Biociências, Porto Alegre, v.5, supl.1, p.609-611, jul. 2007.

16 MANZATTO, C.V.; ASSAD, E.D.; BACCA, J.F.M.; ZARONI, M.J.; PEREIRA, S.E.M. Zoneamento agroecológico da cana-de-açúcar. Rio de Janeiro: Embrapa Solos, 2009. 55 p.

17 MILÊNIA AGROCIÊNCIAS. AMINOL 806. Londrina, 2009. 13 p.

18 BUTIRON. Londrina, 2002. 12 p.

19 HERBIPACK 500 BR. Londrina, 2010. 12 p.

20 HEXARON. Londrina, 2006. 10 p.

21 MILHOME, M.A.L.; SOUZA, D.O.B.; LIMA, F.A.F.; NASCIMENTO, R.F. Avaliação do potencial de águas superficiais e subterrâneas por pesticidas aplicados na agricultura do Baixo Jaguaripe, CE. Engenharia Sanitária Ambiental, v.14 n.3, p.363-372, jul./set. 2009 .

22 MORAES, M.A.D. O mercado de trabalho da agroindústria canavieira: desafios e oportunidades. Economia Aplicada, São Paulo, v.11, n.4, p.605-619, out/dez. 2007.

23 REIS, T.E. da S.; BARROS, O.N.F.; REIS, L.C. Utilização de sistema de informações geográficas para a obtenção de cartas de solo e de declividade do município de Bandeirantes (PR). Revista Geografia, Londrina, v.13, n.1, p. 75-92, jan./ jun. 2004

24 ROSA, F.C.; PINHEIRO, A.; SILVA M.R. Avaliação do potencial de lixiviação de agrotóxicos na bacia do Itajaí. SIMPÓSIO BRASILEIRO DE RECURSOS HÍDRICOS, São Paulo, 2007. Anais ... São Paulo: Associação Brasileira de Recursos Hídricos, 2007. p. 18.

25 SPADOTTO, C.A.; MORAES, D.A.C.; BALLARIN, A.W.; LAPERUTA FILHO, J.; COLENCI, R.A. Araquá: software para avaliação de risco ambiental de agrotóxico. Campinas: Embrapa Monitoramento por Satélite, 2010.

26 TAKAKI, G.M.C.; OKADA, K.; NASCIMENTO, A.E. Contaminação do solo e decomposição de xenobióticos. In: MICROOGANISMOS e agrobiodiversidade o novo desafio para a agricultura. Guaíba: Agrolivros, 2008. p. 441-506. 